\title{
EVALUATION OF GINGIVAL BLEEDING AMONG A GROUP OF CHILDREN AFTER USING NEEM EXTRACT MOUTHWASH VERSUS CHLORHEXIDINE MOUTHWASH: A RANDOMIZED CLINICAL TRIAL
}

Sara Ahmed Mahmoud*, Samar Sherif Ali Mostafa** and Sherif Bahgat El Tawil ${ }^{* * *}$

\begin{abstract}
Background: Gingivitis is primarily caused by dental plaque. Several chemical agents have been incorporated into mouthwashes to guard against plaque accumulation and in the management of gingivitis. Neem was introduced as a herbal alternative treatment of gingivitis with few side effects.
\end{abstract}

Aim: This study aimed to evaluate the gingival bleeding among a group of children suffering from gingivitis after using Neem extract mouthwash versus Chlorhexidine mouthwash.

Participants and Methods: This randomized clinical trial involved 96 children suffering from gingivitis, and aged 8 to 10 years. Children were randomly divided into two equal groups. Group A: used 2\% Neem extract mouthwash. Group B: used 0.125\% Chlorhexidine mouthwash. Sulcus bleeding index (SBI)was recorded before the intervention and after 21 days.

Results: The mean differences in SBI scores between the two treatment groups were statistically significant $(\boldsymbol{p}=0.010)$

Conclusion: Neem extract mouthwash had higher efficacy than Chlorhexidine mouthwash in controlling gingival bleeding and in decreasing SBI scores in the study group. Neem mouthwash could be a very good cost-effective alternative to Chlorhexidine mouthwash.

KEYWORDS: Chlorhexidine, Gingival-Bleeding, mouthwash, Neem-Extract, SBI.

* Associate Professor of Pediatric Dentistry \& Dental Public Health- Faculty of Dentistry- Cairo University

* B.D.S, Faculty of Dentistry, Cairo University, 2007.

** Professor of Pediatric Dentistry \& Dental Public Health Faculty, of Dentistry, Cairo University, Cairo, Egypt. 


\section{INTRODUCTION}

Gingivitis is a highly prevalent situation affecting the periodontal apparatus in pediatric patients. This disease occurs among children and young adolescents as a result of the interaction between bacterial-related factors and the host's limited defense mechanism. ${ }^{1,2}$.

Mechanical plaque control is the corner stone in the prevention of gingival inflammation and is a crucial integrant in its treatment. Despite being a simple and efficient method in the prevention of gingivitis, its effectiveness is affected by the individual's manual dexterity and motivation. Therefore, in addition to mechanical plaque control, the use of antiseptics and therapeutic chemical agents is strongly recommended ${ }^{3}$.

Chlorhexidine ( $\mathrm{CHX})$ is considered the most favored mouthwash. Chlorhexidine has been considered a gold standard. But its long-term usage may cause several side effects such as tooth staining, pigmentation, and taste disturbance. Accordingly, effective herbal substitutes for chlorhexidine with all the satisfactory qualities and without its undesirable effects are highly recommended and have been long-awaited ${ }^{4,5}$.

Neem (Azadirachta indica) has been utilized in India and South Asia for many thousands of years as a good tool for preserving healthy periodontium. Neem has several beneficial properties like antiinflammatory, antiseptic, insecticidal, antiulcer, and other medical properties. It is used for the treatment of periodontitis and other dental diseases. The antibacterial activity of neem has been assessed and known from ancient times. But still, its use for the treatment of gingivitis and periodontitis is not very clear ${ }^{6-8}$

Literature review released very few worldwide in vivo studies assessing the effects of neem extracts on dental plaque acquisition and gingival diseases. Hence, the present study was planned to evaluate the gingival bleeding among a group of children suffering from gingivitis after using Neem extract mouthwash versus Chlorhexidine mouthwash.

\section{PARTICIPANTS AND METHODS}

This study was carried out in the Pediatric Dentistry and Dental Public Health Department, Faculty of Dentistry, Cairo University.

\section{Ethical Aspects:}

Ethical approval, before performing this study was obtained from the "Research Ethics Committee" of the Faculty of Dentistry, Cairo University, with ethical approval number: 4517

This study was registered on ClinicalTrials.gov Identifier: NCT03166163

All guardians received a thorough explanation concerning the clinical procedures as well as the possible risks and informed written consents were obtained from those who approved to enroll their children in the study.

\section{Sample size calculation}

Based on the previous article by Chatterjee et al., ${ }^{6}$ indicated that the percentage of change from baseline in bleeding index among neem is 0.25 and among chlorhexidine is 0.54 . A total sample size of 96 children (48 children per group) was sufficient with power $80 \%$ and $5 \%$ significant level. The sample size was calculated and approved by the Evidence-Based Committee of the Faculty of Dentistry, Cairo University.

\section{Participants}

\section{Eligibility criteria}

Ninety-six healthy and cooperative children, aged from eight to ten years old of both genders who suffered from bleeding on brushing, and by clinical examination, there were bleeding on probing, change in color of gingiva due to inflammation, no evidence of gingival edema, and eruption of upper 
and lower permanent central and lateral incisors, were enrolled within the present study.

Children undergoing orthodontic treatment, using other oral hygiene aids apart from routine teeth brushing, and with a known history of allergy to any mouthwash or drug, or taking antibiotics or antiseptic within the previous two months were excluded from this study.

\section{Randomization}

Randomization was done by computer software "www.random.org".

\section{Blinding}

The examiner, the subject, and the statistician didn't know whether the mouthwash was experimental or control.

\section{Study groups}

Group A: Forty-eight children used 2\% Neem extract mouthwash under parent's supervision twice daily for 21 days.

Group B: Forty-eight Children used 0.125\% Chlorhexidine (ADCO pharmaceutical industry) mouthwash under parent's supervision twice daily for 21 days.

\section{Mouthwashes}

\section{Group A: Neem Extract Mouthwash Preparation}

It was prepared in the laboratories of the Faculty of Pharmacy, Cairo University using the cold maceration technique.

\section{Group B: Chlorhexidine Mouthwash}

Ingredients of chlorhexidine gluconate $0.125 \%$ oral rinse (Arab drug company, Egypt), 0.125\% Chlorhexidine gluconate, in a base containing $11.6 \%$ alcohol, FD\&C Blue No. 1, glycerin, natural and artificial peppermint flavor, PEG-40 sorbitan diisostearate, saccharin sodium, and purified water. Chlorhexidine Gluconate Oral Rinse, $0.125 \%$ is a near-neutral solution ( $\mathrm{pH}$ range 5-7) (www.drugs. com).

\section{Clinical procedure}

Each of the ninety-eight participants was subjected to a comprehensive clinical examination of both soft and hard oral tissues for assessing the health status of the oral region before the beginning of the clinical trial.

At the first visit, after drying the teeth with air using a dental air-water syringe. At baseline (before the study): the Sulcus Bleeding Index ${ }^{12}$ was measured by using the WHO probe (Lite-Touch). The examination was carried out under adequate lighting; the periodontal probe was placed in the gingival sulcus parallel to the long axis of the tooth at the previously mentioned locations for each tooth in all the quadrants and the scores were recorded after 30 seconds ${ }^{13}$. This was done quadrant by quadrant. At the end of the visit, certain instructions were given to the patients including rinsing twice daily in the morning and at evening ( 12 hours interval) by $5 \mathrm{ml}$ mouthwash using graduated Plastic Medicine Cups (Safecare Medical Products Co., Ltd (Hefei), China), group A used 2\% neem extract mouthwash and group B used $0.125 \%$ Chlorhexidine mouthwash that should be supervised by the legal guardians. Kits containing toothpaste and toothbrush were provided ${ }^{14}$. Another visit was scheduled after 3 weeks, the Sulcus Bleeding Index was re-measured and recorded at the end of this clinical trial.

\section{Statistical methods}

Descriptive analysis regarding age was described in terms of mean, standard deviation (SD), while gender is expressed in frequency and percentage. The Kormogrov-simirinov's test for normality was applied. The mean difference ( \pm standard deviation) of sulcus bleeding index scores between groups was calculated. Student's t-test was used to assess the differences between normally distributed data, while, Mann Whitney U test was uses for nonparametric results. The level of significance was set at $\mathrm{p} \leq 0.05^{15}$. 


\section{RESULTS}

The current study was carried out to evaluate the gingival bleeding among a group of children $(n=96$ with an age range from (8-10) years) after using Neem extract mouthwash versus Chlorhexidine mouthwash.

The results of the present study showed that the mean age of the sample subjects in the Neem group was 9.00 years old $( \pm 0.8$ years $)$ which was slightly less than that of the $\mathrm{CHX}$ group which was 9.10 years old $( \pm 0.78$ years). There was no statistically significant difference in mean age between the two groups ( $p$-value $>0.05$ )

Regarding the gender distribution in the study groups, the percentage of male participants in the CHX (control) group subjects was slightly higher $(52 \%)$ than the female percentage (48\%). Within the Neem group, these percentages were reversed (52\% females, $48 \%$ males). Gender mean differences between the two treatment groups were statistically insignificant ( $p$-value $>0.05$ ).

\section{Sulcus Bleeding Index}

The results highlighted that, Sulcus bleeding index mean score of the sample subjects in the Neem group before treatment was $2.38( \pm 0.36)$ which was higher than that of the CHX group which was $1.98( \pm 0.33)$, while Sulcus bleeding index mean score of the sample subjects in the Neem group after treatment was $1.89( \pm 0.32)$ which was higher than that of the CHX group which was $1.73( \pm 0.34)$. The mean difference between scores before and after treatment of the sample subjects in the Neem group was $0.49( \pm 0.43)$ which was higher than that of the CHX group which was $0.26( \pm 0.42)$ as shown in (Table 1). Sulcus bleeding index mean score differences between Neem and Chlorhexidine groups were statistically significant $(p$-value $=0.010)$ as shown in (Table 2).
TABLE (1): Descriptive analysis of the Sulcus Bleeding index treatment groups (Neem and $\mathrm{CHX}$ )- Means, standard deviations, ranges

\begin{tabular}{clccc}
\hline & & Before & After & Diff \\
\hline \multirow{2}{*}{ Neem } & Mean & 2.38 & 1.89 & 0.49 \\
\cline { 2 - 5 } & Median & 2.43 & 1.88 & 0.49 \\
\cline { 2 - 5 } & Std. Deviation & 0.36 & 0.32 & 0.43 \\
\cline { 2 - 5 } & Range & 1.65 & 1.28 & 1.16 \\
\hline \multirow{2}{*}{$\begin{array}{c}\text { CHX } \\
\text { control) }\end{array}$} & Mean & 1.98 & 1.73 & 0.26 \\
\cline { 2 - 5 } & Median & 2.00 & 1.69 & 0.09 \\
\cline { 2 - 5 } & Std. Deviation & 0.33 & 0.34 & 0.42 \\
\hline
\end{tabular}

TABLE (2): Comparison between the differences in Sulcus Bleeding index scores in Neem and Chlorhexidine (control) treatment groups - results of non-parametric Mann Whitney U test (p-value).

\begin{tabular}{ccc}
\hline \multirow{2}{*}{ Teeth } & \multicolumn{2}{c}{ Sulcus Bleeding index } \\
\cline { 2 - 3 } & $\begin{array}{l}\text { Mann Whitney U } \\
p \text {-value* }\end{array}$ & \multicolumn{1}{c}{ Interpretation } \\
\hline \multirow{2}{*}{ All } & 0.010 & $\begin{array}{l}\text { Statistically significant } \\
\text { difference }\end{array}$ \\
\hline
\end{tabular}

*Statistical significance at p-value $\leq 0.05$.

\section{DISCUSSION}

Gingivitis is a common oral disease that starts in early childhood and its severity increases with age. If left untreated, it can result in more progressing the condition to develop periodontal disease ${ }^{16}$.

Chlorhexidine has been utilized for several decades as one of the most effective anti-plaque agents in preventive dentistry. However, long-term use of chlorhexidine is limited due to approved disadvantages. Therefore, new formulations with similar or superior efficacy and possibly fewer longterm effects need to be investigated ${ }^{7,17}$. 
Natural herbs (as Neem extract) have been scientifically proven to be safe against various oral health problems. Neem is of particular interest to the field of dentistry as it has been used in treating teeth and gum problems for a long time ${ }^{8,18}$. Neem herbal extracts elaborate biologically active compounds that have anti-inflammatory, antihyperglycaemic, antiulcer, antimalarial, antifungal, antibacterial, antiviral, antioxidant, antimutagenic, and anticarcinogenic properties and with minimum side effects, therefore was used in the present study ${ }^{8,19}$.

Therefore, the current study was performed to evaluate the effect of Neem extract mouthwash and Chlorhexidine Mouthwash on gingival bleeding among a group of Children. The sulcus bleeding index was used to assess gingival bleeding because it is believed that bleeding from the sulcus is the earliest clinical symptom of gingivitis ${ }^{11,20}$.

Children with fixed orthodontic appliances were excluded as these appliances make the patients vulnerable to plaque accumulation and exaggerate the condition of gingival inflammation and standardize the effect of Chlorhexidine and Neem in all the studied subjects ${ }^{21}$.

Children taking antibiotics in the previous two months and who using any other oral hygiene aid other than routine teeth brushing were also excluded to avoid any interference with the effects of mouthwashes used ${ }^{9,21}$.

Children were instructed to use $5 \mathrm{ml}$ of the assigned mouthwash, two times a day for 21 days. This regimen acquiesces to the standardized protocol of chlorhexidine mouthwash, as it has been proved to suppress salivary bacterial titer for over 12 hours. Kits containing toothpaste and toothbrush were provided so that the patients used the same toothpaste, avoiding any interference with the results ${ }^{4,21}$.

Children aged 8-10 years were included in the study as a mouth rinse is not recommended for younger children who cannot control their swallowing reflex effectively ${ }^{22}$.
Neem mouthwash preparation (2\% concentration) was done according to Sharma et al., ${ }^{4}$ and Balappanavar et al., ${ }^{9}$ so that the taste should not be a hindrance for its use with maximal bacteria and dental plaque inhibition. In this study, Neem sticks were used instead of leaves as it has been proven that neem stick extract had higher antibacterial properties than the leaves extract ${ }^{4,23}$.

An artificial sweetening agent (30\% sucralose) was added to make the taste pleasant. No flavoring agents were added to the extract preparation as they are essential oils and might act as confounders in plaque and gingival assessment ${ }^{4}$.

The preservative agents used in extract preparation (Sodium benzoate and Methylparaben), have long been used as a food preservative, with proven safety, and with antibacterial properties even at lower concentrations ${ }^{4}$.

In the present study, there were non-significant differences in mean age and gender, between Group A (Neem extract) and Group B (Chlorhexidine). Therefore, the two study groups were comparable and had not affected the measured outcome.

Regarding results concerning the efficiency of chlorhexidine in reducing the sulcus bleeding index scores in children, the mean score of the sample subjects in the chlorhexidine group after treatment (1.73) was lower than that before treatment (1.98), (Table 1). These results were following Batista et al., ${ }^{24}$ Sachdeva et al., ${ }^{25}$ and Al-Kamel et al., ${ }^{26}$ who found that chlorhexidine $0.125 \%$ mouthwash was effective in reducing gingival bleeding in periodontal disease through its antibacterial action.

Regarding results concerning the efficiency of Neem in reducing the sulcus bleeding index scores in children, Neem caused a significant reduction in the mean score of the sample subjects from (2.38) before treatment to (1.89) after treatment (Table 1). Similar to that, other studies found that the Neem extract showed the most antimicrobial activity against Streptococcus mutans which is one of the main components of dental plaque, and reduced gingival bleeding 21,27-29. 
Neem extract contains isoprenoids such as azadirachtin, nimbinin and nimbidin as well as chloride and fluoride which exert anti-inflammatory and antibacterial effects against several oral streptococci and reduces bacterial adhesion to the tooth surface, dental plaque accumulation, and gingival bleeding ${ }^{30,31}$.

Contrary to the results of the current study, Sharma et al., ${ }^{32}$ declared that Neem induced a nonsignificant reduction in the level of plaque and gingival indices. This might be due to differences in the study methodology.

Comparing the efficiency of Chlorhexidine and Neem in reducing sulcus bleeding index scores in children, there was a statistically significant difference between them ( $p$-value $=0.010)$ as shown in table 2, where neem was more efficient than chlorhexidine. These results came in agreement with Balappanavar et al., 9 Pai et al., ${ }^{33}$ who found a significant decrease in the plaque index in the group treated with the Neem extract gel than that treated with chlorhexidine mouthwash.

On the other hand, the results of the current study contradict the results of Chatterjee et al., ${ }^{6}$ Singh et al., ${ }^{8}$ Botelho et al., ${ }^{17}$ Pai et al., ${ }^{27}$ Jain et al., ${ }^{34}$ Kankariya et al., ${ }^{35}$ and Mahajan et al., ${ }^{36}$ this may be due to the difference in the concentrations of used neem mouthwash, different substantivity of chlorhexidine and neem extract gel, the wide age range of patients (18-65 years), the neem mouthwash extracted from the plant leaves not the sticks, inadequate spread, and penetration extent of gel's antimicrobial component, their usage on Neem plants in their crude form, and the different study design (in-vitro study) respectively.

\section{CONCLUSION}

This study concluded that Neem extract mouthwash was effective in controlling gingival bleeding on brushing. Moreover, it had higher efficacy than Chlorhexidine mouthwash in decreasing sulcus bleeding index scores in the present study group. Finally, Neem extract mouthwash could be a very good cost-effective alternative to Chlorhexidine mouthwash with fewer side effects.

\section{Recommendations}

Further clinical trials are required to be conducted on different concentrations of Neem extract mouthwash, and for the standardization and preparation of Neem extract mouthwashes for the prevention of oral microbial diseases. More efforts are required to clarify and improve the understanding of the role of this herbal mouthwash in gingival and periodontal disease.

\section{REFERENCES}

1. Gomes C E B, Cavalcante D G, Filho J E, da Costa F N, \& da Silva Pereira S L. Clinical effect of a mouthwash containing Anacardium occidentale Linn. on plaque and gingivitis control: A randomized controlled trial. Indian journal of dental research : official publication of Indian Society for Dental Research, 2016; 27(4): 364-369.

2. Liccardo D, Cannavo A, Spagnuolo G, Ferrara N, Cittadini A, Rengo C, \& Rengo G. Periodontal Disease: A Risk Factor for Diabetes and Cardiovascular Disease. International Journal of Molecular Sciences, 2019; 20: 1414-1428.

3. Rajendiran M, Trivedi H M, Chen D, Gajendrareddy P, \& Chen L. Recent Development of Active Ingredients in Mouthwashes and Toothpastes for Periodontal Diseases. Molecules 2021; 26(7): 2001-2022 https://doi. org/10.3390/molecules 26072001

4. Sharma R, Hebbal M, Ankola AV, Murugaboopathy V, \& Shetty S J. Effect of two herbal mouthwashes on gingival health of school children. Journal of Traditional and Complementary Medicine, 2014; 4(4): 272-278.

5. Kumar K H, Gundala R, Kothari D, Sankar K R, Prasad A S, \& Gowthami N. A Comparative Clinical Evaluation of Multi-Herbal and $0.2 \%$ Chlorhexidine Mouth Wash on Plaque and Gingivitis Control: A Randomized Clinical Trial. International Journal of Advanced Research. 2020; 8(01): 1157-1161.

6. Chatterjee A, Saluja M, Singh N \& Kandwal A. To evaluate the antigingivitis and antipalque effect of an Azadirachta indica (neem) mouthrinse on plaque induced gingivitis: A double-blind, randomized, controlled trial. Journal of Indian Society of Periodontology, 2011; 15(4): 398-401.

7. Safiaghdam H, Oveissi V, Bahramsoltani R, Farzaei MH, Rahimi R. Medicinal plants for gingivitis: a review of 
clinical trials. Iranian Journal of Basic Medical Sciences. 2018;21:978-991. doi: 10.22038/IJBMS.2018.31997.7690

8. Singh V, Pathak A K, Goel K, \& Pal M. Evaluation of Topical Application of Azadirachta indica (Neem) in Prevention of Gingivitis: A Randomized Control Trial. Open Journal of Stomatology, 2019, 9:249-259. https:// doi.org/10.4236/ojst.2019.911026

9. Balappanavar AY, Sardana V \& Singh M. Comparison of the effectiveness of $0.5 \%$ tea, $2 \%$ neem and $0.2 \%$ chlorhexidine mouthwashes on oral health: a randomized control trial. Indian Journal of Dental Research: Official Publication of Indian Society for Dental Research, 2013; 24(1): 26-34.

10. Jalaluddin M, Rajasekaran UB, Paul S, Dhanya RS, Sudeep CB \& Adarsh VJ. Comparative evaluation of neem mouthwash on plaque and gingivitis: A double-blind crossover study. Journal of Contemporary Dental Practice, 2017; 18(7): 567-571.

11. Grellmann A P \& Zanatta F B. Diagnosis of gingivitis: State of the Art. Journal of Dentistry \& oral disorders, 2016; 2(3): 1-8.

12. Mühlemann HR \& Son S. Gingival sulcus bleeding-a leading symptom in initial gingivitis. Helvetica Odontologica Acta, 1971 Oct; 15(2): 107-113.

13. Muthu M S \& Sivakumar N. Pediatric Dentistry Principles and practice. ELSEVIER, India, 2011, Second edition, 497-498.

14. Newman M G, Takei H, Kokkevold P R \& Carranza F A. Carranza's clinical periodontology. Saunders, 2012, eleventh edition, 254-255.

15. R statistical package, version 3.3.1 (2016-06-21). Copyright (C) 2016. The R Foundation for Statistical Computing.

16. Kaur A, Gupta N, Baweja D.K \& Simratvir M. An epidemiological study to determine the prevalence and risk assessment of gingivitis in 5-, 12- and 15-year-old children of rural and urban area of Panchkula (Haryana). Indian Journal of Dental Research: Official Publication of Indian Society for Dental Research, 2014; 25(3): 294-299.

17. Botelho M A, dos Santos R A, Martins J G, Carvalho C O, Paz M C, Azenha C, \& Ruela F I. Efficacy of a mouthrinse based on leaves of the neem tree (Azadirachta indica) in the treatment of patients with chronic gingivitis: A doubleblind, randomized. controlled trial. Journal of Medicinal Plants Research, 2008; 2(11): 341-346.
18. Mahajan R, Khinda P, Gill A, Kaur J, Saravanan S, Sahewal A \& Joshi, V. Comparison of Efficacy of $0.2 \%$ Chlorhexidine Gluconate and Herbal Mouthrinses on Dental Plaque: An in vitro Comparative Study. European Journal of Medicinal Plants, 2016; 13(2): 1-11.

19. Lakshmi T, Krishnan V, Rajendran R \& Madhusudhanan N. Azadirachta indica: A herbal panacea in dentistry - An update. Pharmacognosy Reviews, 2015; 9(17): 41-44.

20. Panagakos F S \& Davies R M. Gingival Diseases-Their Aetiology, Prevention and Treatment, InTech, Croatia, 2011, Frist edition.

21. Nishad A, Sreesan N S, Joy J, Lakshmanan L, Thomas J \& Anjali V A. Impact of Mouthwashes on Antibacterial Activity of Subjects with Fixed Orthodontic Appliances: A Randomized Clinical Trial. The Journal of Contemporary Dental Practice, 2017, 18(12): 1112-1116.

22. Harmbire C U, Jawade R, Patil A, Wani V R, Kulkarni A A \& Nehete P B. Comparing the antiplaque efficacy of $0.5 \%$ camellia sinensis extract, $0.05 \%$ sodium fluoride, and $0.2 \%$ chlorhexidine gluconate mouthwash in children. Journal of Internal Society of Preventive \& Community Dentistry, 2015; 5(3): 218-226.

23. Siswomihardgo W, Badawi S S, Nishimura M, \& Hamada T. The difference of antibacterial effect of neem leaves and stick extracts. International Chinese Journal of Dentistry, 2007; 7: 27-29.

24. Batista A L, Diógenes R, Uchôa A, Coelho R D S, Barbosa $\mathrm{N}$ \& Celestino A. Complementary Therapies in Clinical Practice Clinical efficacy analysis of the mouth rinsing with pomegranate and chamomile plant extracts in the gingival bleeding reduction. Complementary Therapies in Clinical Practice, 2014; 20(1): 93-98.

25. Sachdeva S, Grover V, Malhotra R \& Kapoor A. Comparison of clinical effectiveness of single and multiple applications of $1 \%$ chlorhexidine varnish ( Cervitec Plus ) along with scaling and root planing in patients with chronic periodontitis. Journal of Indian Society of Periodontology, 2018 Nov-Dec; 22(6): 523-528.

26. Al-kamel A, Al-hajj W A, \& Halboub E. N-acetyl cysteine versus chlorhexidine mouthwashes in prevention and treatment of experimental gingivitis: a randomized, tripleblind, placebo-controlled clinical trial. Clinical Oral Investigations, 2019 Jan 23; 23(1): 1-10.

27. Pai M.R, Acharya L.D \& Udupa N. Evaluation of antiplaque activity of Azadirachta indica leaf extract gel - A 6-week clinical study. Journal of Ethnopharmacology, 2004; 90(1): 99-103. 
28. Elangovan A, Muranga J \& Joseph E. Comparative evaluation of the antimicrobial efficacy of four chewing sticks commonly used in South India: An in vitro study. Indian Journal of Dental Research 2012; (23)6: 840-842.

29. Abhishek K.N, Supreetha S, Sam G, Khan S.N, Chaithanya K.H \& Abdul, N. Effect of Neem containing Toothpaste on Plaque and Gingivitis: A Randomized Double Blind Clinical Trial. The Journal of Contemporary Dental Practice, 2015 ; 16(11): 880-883.

30. Kaushik A, Kaushik M, \& Tanwar R. Ethnomedicine: Applications of Neem (Azadirachta indica) in dentistry. Dental Hypotheses, 2012; 3(3): 112-114.

31. Lakshmi T, Krishnan V, Rajendran R \& Madhusudhanan N. Azadirachta indica: A herbal panacea in dentistry - An update. Pharmacognosy Reviews, 2015; 9(17): 41-44.

32. Sharma S, Saimbi C, Koirala B \& Shukla R. Effect of Various Mouthwashes on the Levels of Interleukin-2 and Interferon- $\gamma$ in Chronic Gingivitis. Journal of Clinical Pediatric Dentistry, 2007, 32(2): 111-114.
33. Pai M R, Acharya L D \& Udupa N. The effect of two different dental gels and a mouthwash on plaque and gingival scores: a six-week clinical study, International Dental Journal, 2004 Aug; 54(4): 219-23.

34. Jain I, Jain P, Bisht D, Sharma A, Srivastava B \& Gupta N. Use of Traditional Indian Plants in the Inhibition of CariesCausing Bacteria - Streptococcus mutans. Brazilian Dental Journal. 2015; 26(2): 110-115.

35. Kankariya A R, Patel A R \& Kunte S S. The effect of different concentrations of water soluble azadirachtin (neem metabolite) on Streptococcus mutans compared with chlorhexidine. Journal of Indian Society of Periodontology Preventive Dentistry. 2016 Apr-Jun; 34(2): 105-110.

36. Mahajan R, Khinda P, Gill A, Kaur J, Saravanan S, Sahewal A \& Joshi, V. Comparison of Efficacy of $0.2 \%$ Chlorhexidine Gluconate and Herbal Mouthrinses on Dental Plaque: An in vitro Comparative Study. European Journal of Medicinal Plants, 2016; 13(2): 1-11. 